\title{
Newer Trial Designs
}

\author{
Azgar Abdul Rasheed ${ }^{1}$ Venkata Pradeep Babu Koyyala²
}

\author{
${ }^{1}$ Department of Medical Oncology, Dr. BRA IRCH, AIIMS, \\ New Delhi, India \\ 2Department of Medical Oncology, Rajiv Gandhi Cancer Institute \\ and Research Centre, New Delhi, India
}

Ind J Med Paediatr Oncol 2021;42:96-98.

Clinical trials are vital to the discovery, development, and testing of new therapies. In the early years of oncology, clinical trials were conducted based on the cancer type and stage. Later on, we moved on to "enrichment'7" targeted" designs for the evaluation of clinical utility, choosing patient populations according to a single-molecular marker, such as epidermal growth factor receptor (EGFR) in head-and-neck squamous cell carcinoma, anaplastic lymphoma kinase (ALK) in nonsmall cell lung cancer (NSCLC), or human epidermal growth factor receptor 2 mutations in breast cancer. This helped to select the study populations in which each drug was more likely to be effective compared with an unselected population. To validate the clinical utility of a marker and determine if it is worth studying further, "Marker by Treatment Interaction" or "Marker-based Strategy" designs can be used (-Figs. 1 and 2). In the marker by treatment-interaction design, the marker is measured in all patients, who are then stratified by their level of marker expression. In each group, the patients will be assigned different therapies to see if one treatment is superior to another. It is equivalent to doing two or more randomized controlled trials (RCTs) (e.g., two RCTs if the population is stratified into marker positive and marker negative groups). In the marker-based strategy design, after measuring the marker in all patients, they are randomized into two arms: In one, the treatment is decided according to marker status: standard therapy for marker-negative patients and experimental therapy for marker-positive patients. In the other arm, the treatment may be independent of marker status, with all receiving standard therapy or the patients may again be randomized to standard versus experimental therapy, irrespective of marker status. For a useful marker, the treatment outcomes in the marker-dependent treatment arm should be at least $30 \%$ better than in the marker-independent treatment arm.

At present, technological advances have enabled the characterization of tumors in ever greater detail, and consequently, we are faced with a higher number of precise targets
Address for correspondence Venkata Pradeep Babu Koyyala, MD, DNB Medical Oncology Department of Medical Oncology, Rajiv Gandhi Cancer Institute and Research Centre 110085, New Delhi, India (e-mail: pradeepbabu.koyyala@gmail.).

against that new therapeutic interventions can be developed. It has even become possible to classify the tumors by molecular subtypes, regardless of their tissue of origin, such as the breast, colorectal, or lung. Since it would be impractical to design and conduct the separate clinical trials for each of these subpopulations, we now employ "master protocol" trial designs.

What is a "master protocol?" A master protocol is a single, overarching protocol, from which we can derive subprotocols for multiple substudies that run simultaneously. Each substudy targets a specific-molecular marker or therapy. Having a master protocol avoids wasting repetitive efforts on design, regulatory approval, and logistics planning for each trial. Master protocol trials can be "exploratory" phase I/II or "confirmatory" randomized phase III trials. Basket, umbrella, and platform designs are different kinds of master protocol trials, although each does not have a standardized definition. The advantage of master protocol trials is that they enable the concurrent testing of a wide range of markers, while overcoming heterogeneities in the patient population and reducing costs. One of the important drawbacks is a possible increase in false-positive findings due to concurrently running several small substudies.

\section{Basket Trials/Bucket Trials}

Basket trials ( - Fig. $\mathbf{3}$ ) are groups of exploratory, single-arm, phase II trials aimed at "signal-finding" or selecting drugs for further development. They are tissue-agnostic or histology-independent studies, with each "basket" or substudy representing a collection of patients having a specific marker in common, even if their tumors have different histological origins. Each substudy usually has 20 to 50 patients and tests a particular-targeted therapy against the mutation in that basket. The primary end point in these studies is often the response rate. Because of the small sample sizes and the lack of comparator arms, these trials are not useful
DOI https://doi.org/ 10.1055/s-0041-1729435 ISSN 0971-5851

\section{(c) 2021. Indian Society of Medical and Paediatric Oncology}

This is an open access article published by Thieme under the terms of the Creative Commons Attribution-NonDerivative-NonCommercial-License, permitting copying and reproduction so long as the original work is given appropriate credit. Contents may not be used for commercial purposes, or adapted, remixed, transformed or built upon. (https://creativecommons.org/licenses/by-nc-nd/4.0/).

Thieme Medical and Scientific Publishers Pvt. Ltd. A-12, 2nd Floor, Sector 2, Noida-201301 UP, India 
Marker x Treatment Interaction Design

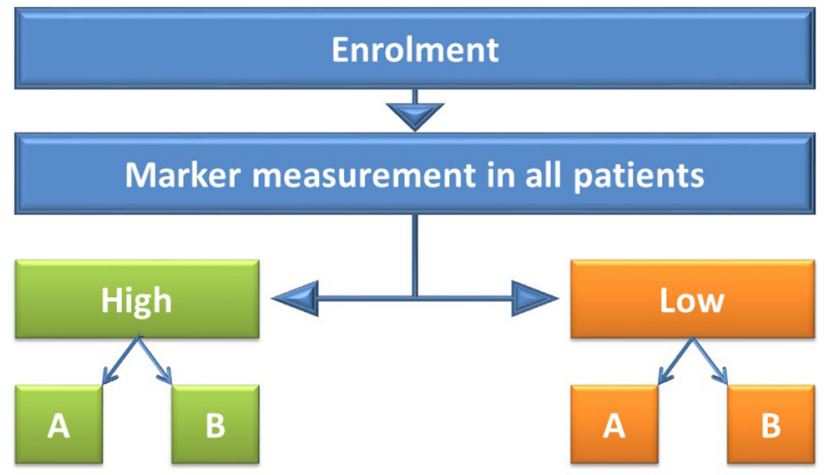

Fig. 1 Marker $\mathrm{x}$ treatment interaction design.

\section{Marker Strategy Design}

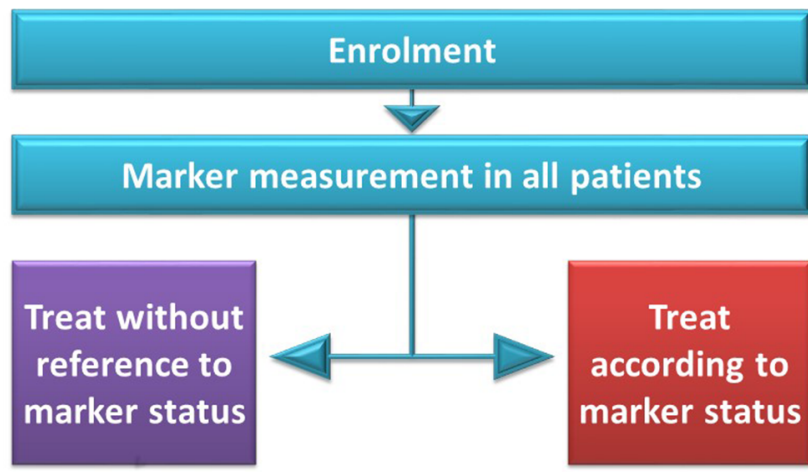

Fig. 2 Marker strategy design.

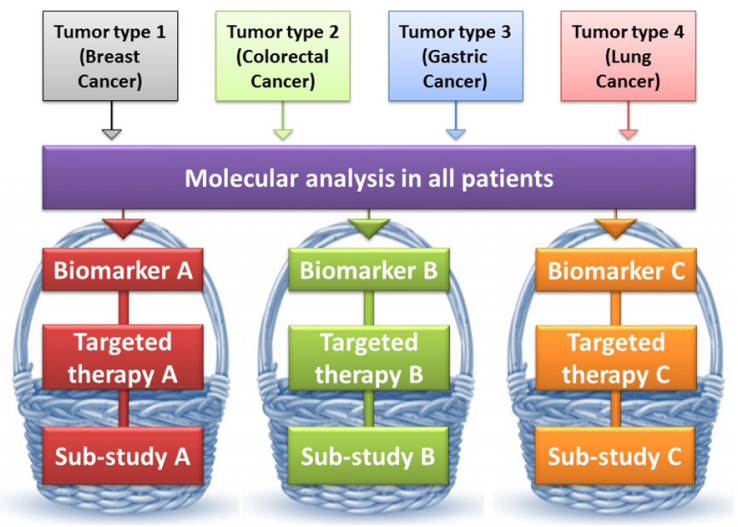

Fig. 3 Basket trial design.

for establishing the efficacy of a drug and may even fail to differentiate between the predictive and prognostic effects of a marker. Their utility lies in efficiently identifying the effective treatments for cancers at multiple sites with a common genetic mutation, and the cross testing of drugs approved in one histology to other diseases. An example of this design is a phase II "basket" study of vemurafenib in rapid acceleration of fibrosarcoma gene B (BRAF) V600 mutation-positive nonmelanoma cancers by Hyman et al. ${ }^{1}$ They enrolled 122 patients with colorectal, breast, ovarian, or NSCLC, cholangiocarcinoma, or Langerhans'-cell histiocytosis, all having a mutation in BRAF V600. Response rate was the primary end point, with progression-free survival and overall survival (OS) as the secondary end points. An important aspect to consider when interpreting basket trials is that the microenvironment of a tumor will also dictate its response to therapy, not just the mutation profile, and we do not have enough evidence at this point to conclude that molecular makers should replace histology-based tumor typing.

\section{Umbrella Trials}

In umbrella trials or "molecular allocation studies," substudies are conducted testing multiple targeted therapies against different molecular markers in the same tumor (-Fig. 4). Hence, the tumor type/histology is the "umbrella" covering all the substudies. These can vary from single-arm, phase II, to randomized, phase II/III trials with placebo or standard therapy arms. Because of the difficulty in getting adequate patients of each molecular type, umbrella trials are usually long-term studies and large in scale. If a patient is eligible for more than one substudy, the overlap is dealt with in the statistical analysis. The advantages of this design are its usefulness in testing markers with a low prevalence, and the confirmatory nature of these trials, enabling the generation of high-level evidence for the therapies they test. A typical example of this sort of design is the Adjuvant Lung Cancer Enrichment Marker Identification and Sequencing Trials (ALCHEMIST), ${ }^{2}$ a set of RCTs in early-stage NSCLC patients. Post complete resection of the primary tumor, EGFR-positive patients (10-15\%) are enrolled in a phase III randomized substudy comparing erlotinib versus placebo and ALK-positive patients (5-6\%) to one comparing crizotinib versus placebo. Those negative for both, depending on their programmed

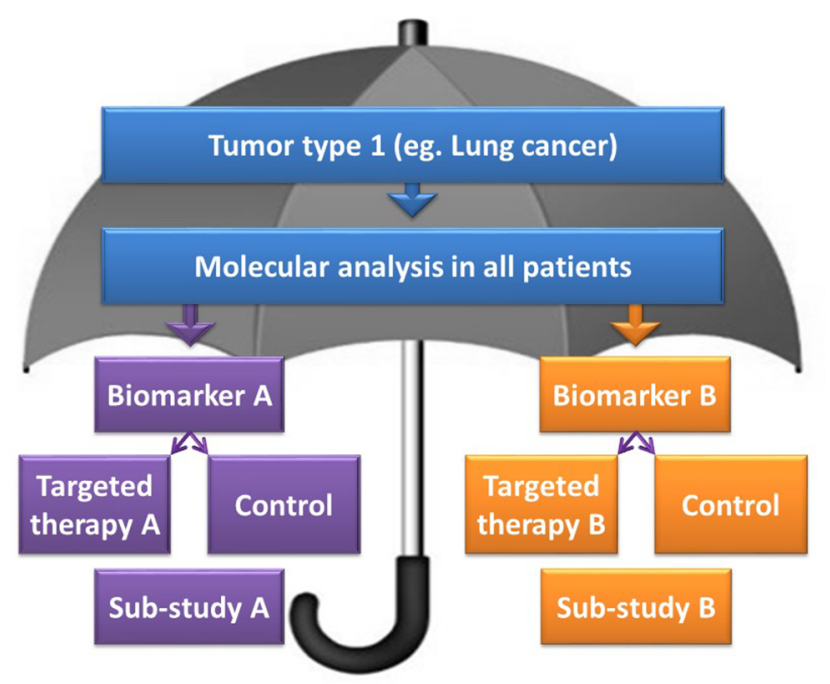

Fig. 4 Umbrella trial design. 
death ligand 1 expression, may be enrolled in a randomized substudy comparing nivolumab versus observation. The end point of all these substudies is OS.

"Super Umbrella Trials" are a combination of bucket trials and umbrella trials. They can be thought of as umbrella trials in which tumors of different histologies can be enrolled. An example is the National Cancer Institute Molecular Analysis for Therapy Choice (MATCH) trial, ${ }^{3}$ which covered 18 biomarkers, 12 different treatments, and patients with both common and rare cancers.

\section{Adoptive Platform Trials}

Adaptive platform trial are significantly different from traditional RCTs. ${ }^{4}$ They are designed to run perpetually, testing multiple-targeted therapies one after the other. Varied treatments and patient populations can enter or leave the platform during the lifetime of the trial, as determined by a decision algorithm. The substudies are continually modified based on the results of preplanned interim analyses performed to evaluate the futility or efficacy of each targeted therapy. Theseadaptations, suchas response-adaptive randomization, result in the modification of treatment assignments, addition/termination of study arms, and the transitioning of the trials from one phase to the next. These adaptations can be retrospective, concurrent, or prospective. Like umbrella trials, these too are large-scale, long-term studies, and expensive to execute. However, they require fewer patients overall, have faster accrual, and are more efficient than classical clinical trial designs. One such trial is the I-SPY2 (Investigation of Serial studies to Predict Your therapeutic response with imaging and molecular analysis 2) study, which has been continually running since 2010. It is an open-label phase II trial comparing the efficacy of a combination of experimental and standard chemotherapy drugs versus standard chemotherapy alone, for women with newly diagnosed, locally advanced breast cancer (stage II/III), before surgical resection. Pathological complete response is the primary outcome.

\section{Financial Support and Sponsorship}

Nil.

\section{Conflicts of Interest}

There are no conflicts of interest.

\section{References}

1 Hyman DM, Puzanov I, Subbiah V, et al. Vemurafenib in multiple non-melanoma cancers with BRAF $\mathrm{V} 600$ mutations. N Engl J Med 2015;373(8):726-736

2 The ALCHEMIST Lung Cancer Trials - National Cancer Institute. Available from: https://www.cancer.gov/types/lung/rese arch/ alchemist. Accessed on April 7, 2021.

3 Flaherty KT, Gray R, Chen A, et al. NCI-MATCH Team. The molecular analysis for therapy choice (NCI-MATCH) trial: lessons for genomic trial design. J Natl Cancer Inst 2020;112(10):1021-1029

4 Angus DC, Alexander BM, Berry S, et al. Adaptive Platform Trials Coalition. Adaptive platform trials: definition, design, conduct and reporting considerations. Nat Rev Drug Discov 2019;18(10):797-807 